\title{
INDIVIDUAL MOTIONS OF RED BLOOD CELLS IN HIGH-HEMATOCRIT BLOOD FLOWING IN A MICROCHANNEL WITH COMPLEX GEOMETRIES
}

\author{
T. Ishikawa ${ }^{1}$, H. Fujiwara ${ }^{1}$, N. Matsuki ${ }^{2}$, R. Lima ${ }^{3}$, Y. Imai ${ }^{1}$, H. Ueno ${ }^{4}$ and T. Yamaguchi ${ }^{2}$
}

\author{
*1 Dept. Bioeng. Robotics, Tohoku University, 6-6-01 Aoba, Aoba-ku, Sendai 980-8579, Japan \\ ${ }^{2}$ Dept. Biomed. Eng., Tohoku University, 6-6-01 Aoba, Aoba-ku, Sendai 980-8579, Japan \\ ${ }^{*}$ Dept. Mech. Tech., Braganca Polytechnic Inst., C. Sta. Apolonia, Braganca 5301-857, Portugal \\ ${ }^{\star 4}$ Tohoku University Int. Adv. Res. Edu., 6-6-01 Aoba, Aoba-ku, Sendai 980-8579, Japan
}

\section{INTRODUCTION}

Blood flow in a microchannel with complex geometries has been investigated to develop biomedical microdevices (e.g. Faivre et al., 2006) or to understand pathology in small vessels, such as lacunar infarcts. In a small channel, say $100 \mu \mathrm{m}$ in diameter, the blood is no longer assumed to be a homogeneous fluid because the size of the red blood cells (RBCs) cannot be neglected compared to the generated flow field (the diameter of a RBC is about $8 \mu \mathrm{m}$ ). In such a case, we must treat the blood as a multiphase fluid, and investigate the motion of individual cells in discussing the flow field. In this study, we investigated the motion of RBCs in a microchannel with stenosis or bifurcation using a confocal micro-PTV system. We measured individual trajectories of RBCs under high Hct conditions (up to 20\%), when the interactions between RBCs become significant. We discuss the effect of Hct on the flow field and cell-free layers, as well as the effect of deformability of RBCs on the cell-free layer thickness by hardening RBCs using a glutaraldehyde treatment.

\section{MATERIALS AND METHODS}

\subsection{Confocal micro-PTV system}

The confocal micro-PTV system used in this study was the same as that reported by Lima et al. (2008). Briefly, it consisted of an inverted microscope, a confocal scanning system, a high-speed camera, a DPSS laser, a syringe pump for making constant flow, a thermo plate for controlling the temperature, and an objective lens (x20). The estimated thickness of the measurement plane was 4.97 $\mu \mathrm{m}$. By exposing the laser to the labeled RBCs, the system enabled us to track individual RBCs inside the blood flow of up to $20 \%$ Hct with high resolution and low optical thickness. The recorded images were evaluated in Image $\mathbf{J}$ using the manual tracking MtrackJ plug-in.

\subsection{Materials}

Microchannels were made of polydimethylsiloxane (PDMS). A microchannel with stenosis had a square cross section with a side length of $50 \mu \mathrm{m}$, and the stenosis was $35 \mu \mathrm{m}$ high and $30 \mu \mathrm{m}$ wide. The stenosis was $50 \mu \mathrm{m}$ in depth, so the cross section of the channel in the $x-y$ plane did not vary in the $z$-direction. In the case of a microchannel with bifurcation, the parent channel had $75 \mu \mathrm{m}$ wide and $40 \mu \mathrm{m}$ depth, and the daughter channels had $50 \mu \mathrm{m}$ wide and $40 \mu \mathrm{m}$ depth. The angle between the daughter channels was $60^{\circ}$.

Dextran 40 (DEX40; 10\% w/w) was used as a solvent fluid of RBCs. RBCs were labeled with a fluorescent dye (C-7000). A glutaraldehyde treatment was used to harden RBCs. The RBCs were taken from a 23-year-old male volunteer and centrifuged to separate the RBCs and plasma completely. Then, they were preserved at $4^{\circ} \mathrm{C}$ and dispersed in pure saline prior to the experiment. All procedures were carried out in compliance with the Ethics Committee on Clinical Investigation of Tohoku University.

\subsection{Experimental conditions}

The average velocity was maintained at about $0.45 \mathrm{~mm} / \mathrm{s}$. The microchannel was surrounded by adiabatic walls, and the temperature of the channel was maintained at $37^{\circ} \mathrm{C}$. The frame rate for the highspeed camera was 300 frames per second.We observed the motion of RBCs in the center plane, i.e. $25 \mu \mathrm{m}$ from the bottom for the stenosed channel and $20 \mu \mathrm{m}$ from the bottom for the bifurcation channel.

\section{RESULTS IN A MICROCHANNEL WITH STENOSIS}

\subsection{Flow of pure water}

First, we investigated the flow of pure water. The trajectories of tracer particles were measured by continuously tracking individual 
particles. We observed that the trajectories were almost symmetric between the upstream and the downstream of the stenosis. This result is consistent with the Stokes flow condition, where the streamlines become symmetric if the stenosis shape is symmetric to the $y$-axis.

\subsection{Flow of $10 \%$ Hct blood with healthy RBCs}

Next, labeled RBCs in the blood flow with $10 \%$ Hct were visualized at the center plane. The trajectories of individual RBCs were measured by continuous tracking, and the results are shown in Fig. 1(a). The trajectories of RBCs were no longer symmetric. RBCs located initially near the lower wall tended to move away from the wall beyond the stenosis. RBCs located initially near the upper wall, however, again tended to move away from the wall beyond the stenosis. The $y$ position of RBCs changed considerably in the blood flow through the stenosed microchannel.

The asymmetry of the trajectories was induced by the deformation of RBCs. RBCs are deformed by the traction forces generated by the inner- and outer-fluid of the membrane, which depends on the history of deformation along the trajectories. Thus, the deformation of a RBC becomes asymmetric before and after the stenosis, even though the flow field is governed by the Stokes equation.

\subsection{Flow of $20 \%$ Hct blood with healthy RBCs}

We increased the Hct to $20 \%$ and measured the trajectories of labeled RBCs in a blood flow. The results are shown in Fig. 1(b). We observed that the trajectories were not as asymmetric as those at $10 \%$ Hct. To examine this tendency quantitatively, we measured the RBC displacement in the $y$-direction induced by the flow through stenosis. We define $\Delta Y$ equal to $Y_{\text {downstream }}-Y_{\text {upstream, }}$ where $Y_{\text {up stream }}$ and $\mathrm{Y}_{\text {downstream }}$ are the $y$ position of a labeled RBC $60 \mu \mathrm{m}$ upstream and downstream of the stenosis, respectively. The results at $10 \%$ and $20 \%$ Hct with various $Y_{\text {upstream }}$ are shown in Fig. 2. In the case of $10 \%$ Hct, RBCs near the lower wall showed a significant displacement away from the wall. RBCs near the upper wall also showed displacement away from the wall. These results indicate that the RBCs tend to flow in the core region of the bulk flow after flowing through the stenosis, although RBCs initially located in the core region do not move significantly in the $y$-direction. In the case of $20 \% \mathrm{Hct}$, however, the absolute value of $\Delta \mathrm{Y}$ was much smaller. When Hct was high, the interactions between RBCs increased considerably. Thus, the RBCs cannot concentrate in the core region, but are pushed away from the highly concentrated region due to their interactions.

\subsection{Flow of $10 \%$ Hct blood with hardened RBCs}

In order to clarify the effect of cell deformability on the blood flow, we investigated the blood flow with hardened RBCs. Here, we measured the cell-free layer on the upper and lower walls, downstream of the stenosis. We found that the healthy RBCs showed a considerably thicker cell-free layer compared to the hardened RBCs. If $\mathrm{RBCs}$ are hardened, the asymmetry in trajectories is no longer enhanced by the deformation of RBCs. Thus, hardened RBCs initially flowing near the wall can stay near the wall, even after flowing through the stenosis.

\section{RESULTS IN A MICROCHANNEL WITH BIFURCATION}

We also performed similar experiments in a microchannel with bifurcation. The trajectories of RBCs around the bifurcation is shown in Fig. 3 (3\% Hct). We see that RBCs can come very close to the inner wall of the bifurcation, but cannot to the outer wall. The trajectories of RBCs became again asymmetric between the bifurcation and the confluence, though the channel geometry was symmetric and the flow was Stokesian.

\section{CONCLUSIONS}

The present results indicated that deformability has a considerable effect on the asymmetry of the cell-free layer thickness. Therefore, the motions of RBCs are influenced strongly by the Hct, the deformability, and the channel geometry. The results presented here provide fundamental knowledge for a better understanding of blood flow in microcirculations and biomedical microdevices.

\section{ACKNOWLEDGMENTS}

This study was supported by Grant-in-Aid for Scientific Research (S) from JSPS and by Tohoku University Global COE Program

\section{REFERENCES}

Faivre, M. et al. (2006), Biorheology, 43, pp.147-159

Lima, R. et al. (2008) J. Biomech., 41, pp.2188-2196

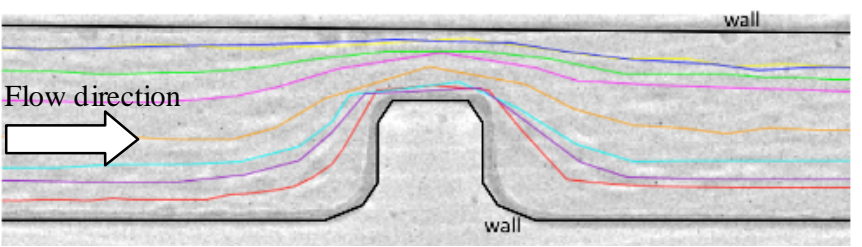

(a) $10 \% \mathrm{Hct}$

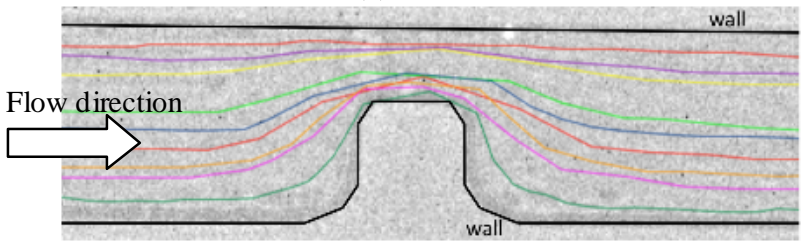

(b) $20 \% \mathrm{Hct}$

Fig. 1. Trajectories of labeled RBCs around the stenosis

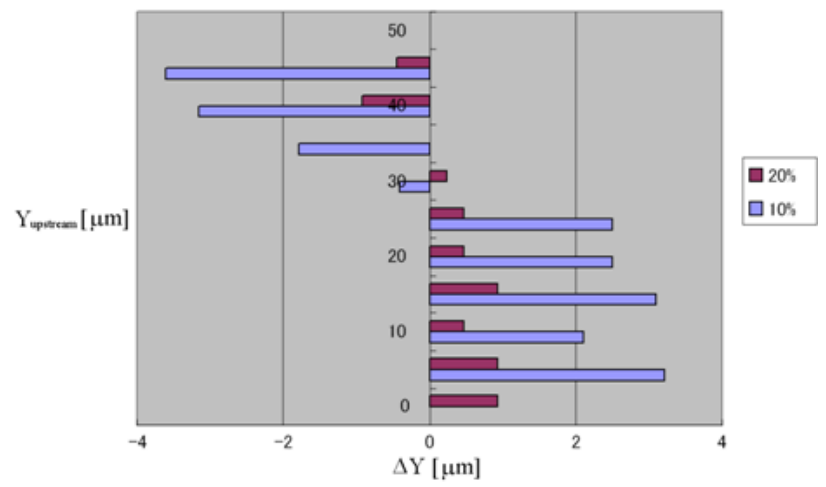

Fig. 2 Effect of Hct and $\mathrm{Y}_{\text {upstream }}$ on $\Delta \mathrm{Y}$.

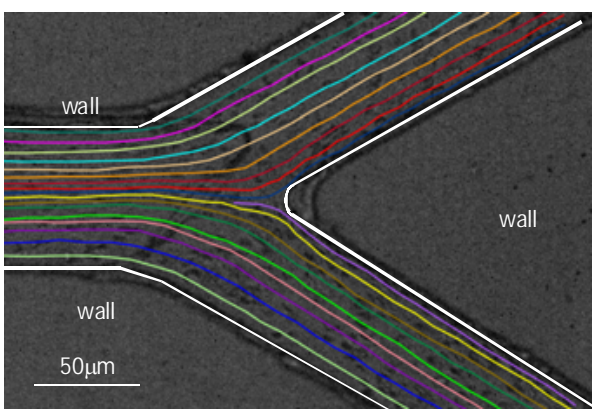

Fig.3. Trajectories of labeled RBCs around the bifurcation (Hct3\%) 